\title{
Electromagnetic Interference in Wireless Communications: Behavioral-Level Simulation Approach
}

\author{
Sergey Loyka \\ School of Information Technology and Engineering (SITE), \\ University of Ottawa, 161 Louis Pasteur, Ottawa, \\ Ontario, Canada, K1N 6N5 E-mail: sergey.loyka@ieee.org
}

\begin{abstract}
Main challenges in electromagnetic interference (EMI) modeling and simulation for modern and future wireless communication systems and networks taking into account nonlinear interference effects are discussed in this paper. It is emphasized that there exists a big difference between simulating analog and digitally-modulated systems when spurious nonlinear effects in the hardware are included. A computationally-efficient approach for the EMI analysis of wireless systems and networks by behavioral-level numerical simulation is proposed. Rapid growth of PCs' computational power allows to implement fullscale simulation of modern wireless systems and networks at the behavioral level at reasonable cost. This technique can be used for simulating optical communication systems as well.
\end{abstract}

Keywords- nonlinear effects, behavioral-level simulation, wireless communications

\section{INTRODUCTION}

Wireless communication systems experience today unprecedented growth. In view of very limited spectrum available and large concentration of system over limited space (dense urban or indoor environment), the potential for mutual interference is tremendous. The large-scale introduction of broadband wireless systems (i.e., 3G), which is foreseen for near future, will make this problem even more important and more difficult to solve due to several reasons: (1) the larger bandwidth of the system results in more interference produced and makes the system more susceptible to external interference, (2) smart antennas, which is a key component in $3 \mathrm{G}$ systems [1,2], are more susceptible to interference due to active circuitry in their structure [3], (3) MIMO architecture, which is very likely to be implemented in the near future due to its unprecedented spectral efficiency [4], makes use of multiple transmit (Tx) and receive $(\mathrm{Rx})$ branches and, hence, produces and receives much more interference, (4) more highquality services offered by such systems at reasonable price will likely increase the number of subscribers and, hence, the number of systems in limited space and spectrum available.

In this paper, we discuss main challenges in EMI modeling and simulation for wireless communication systems, main differences between EMI analyses for analog and digital systems and propose a computationally-efficient approach for system (behavioural) -level simulation of wireless systems at the physical layer (i.e., taking into account almost all the hardware imperfections) which can be efficiently combined with the existing methods of simulation at the signal processing level providing in this way a technique for the simulation of the entire communication link, starting for the source of information and up to the destination point. The emphasis of the paper is on efficient and accurate simulation of nonlinear effects in modern wireless systems. The computational efficiency of the approach proposed is high enough to allow the simulation of real-world systems and even networks under realworld conditions in reasonable time and using reasonable computational resources (i.e., PCs rather than supercomputers).

\section{EMI EFFECTS IN ANALOG AND DIGITAL WIRELESS SYSTEMS}

The mutual interference between wireless systems is presently taken into account mainly during the frequency planning stage and in an oversimplified way, i.e. no comprehensive Tx and Rx models are employed, only few basic parameters (carrier frequency, bandwidth, Tx power, Rx sensitivity and selectivity etc.) are accounted for. The main reason for this is that there is no accurate and computationally-efficient technique available for more accurate and comprehensive analysis. Thus, even carefully made frequency planning does not guarantee the absence of EMI in any scenario.

In general, all the interference effects may be divided into two large groups: (1) linear effect and (2) nonlinear effect. Modeling and simulation of linear effects in communication systems do not present a serious problem and can be carried out in a conventional way [9-11]. On the contrary, accurate modeling and simulation of nonlinear effects in modern communication systems is a big challenge [5]. Thus, we concentrate our discussion on modeling and simulation of nonlinear effects.

Some possible types of nonlinear interference and distortions for wireless communication systems are the following:

1. Spurious radiation of transmitters: harmonics and subharmonics, intermodulation products (IMP), noise etc. Note that the intermodulation radiation can be caused by an external signal (from a nearby transmitter) as well as by several carriers (in CDMA systems, for example) in the transmitter's power amplifier.

2. Spurious responses of receivers: adjacent, image and intermediate frequency (IF) channels. 
3. Nonlinear behavior of receivers may also cause the system performance degradation (desensitization, IMPs, local oscillator noise and harmonics' conversion etc.).

Analytical and semi-empirical methods were extensively used in the past for the analysis of these effects. Those methods were quite simple and allowed one to get some insight into the system operation. However, the accuracy of those methods is rather poor. Besides, the analysis of complex systems (presentday digital wireless systems) is rather difficult if not possible at all to do by the old methods.

Digital communication systems present additional challenge for the EMC/EMI analysis because computational resources required for such an analysis is much higher for digital systems than for analog ones [5]. For example, for a RF power amplifier employed in an analog system, IMP levels present its important performance parameter (among others). The traditional two-signal simulation is sufficient to predict this performance parameter. On the contrary, if this amplifier is employed in a digital system, other performance measures are used - adjacent channel power ratio (ACPR), error vector magnitude (EVM) etc., because the traditional analog parameters (i.e., IMPs, IPs etc.) do not represent adequately the digital system performance [5]. In order to predict these new parameters, multi-tone simulation should be used (typically, with few thousand or even tens of thousand tones), which is much more computationally-expensive. A similar set of new parameters should be employed to predict adequately the performance of digital wireless receivers.

The considerations above suggest that the only likely candidate for the EMC/EMI analysis of present-day and future wireless systems is a numerical simulation approach. A systemlevel analysis, which is required in our case, cannot be done at the circuit-level due to high complexity of the wireless system and of the digitally modulated signals. Besides, the circuit-level simulation would require too much characterization data. Thus, behavioral-level techniques (also known as "black-box" techniques) should be used for such a problem. We further discuss one such technique - the instantaneous quadrature modeling technique.

\section{Quadrature Modeling TeChNiQue}

The quadrature modeling technique is the most popular tool for behavioral-level nonlinear modeling and simulation of the active stages of communication circuits and systems [5, 9]. This technique was introduced in early 1970s for nonlinear modeling and simulation of traveling wave tube amplifiers used in satellite communications. The main idea of the quadrature modeling technique is the use of a complex envelope instead of real narrowband signals:

$$
\begin{aligned}
x(t)= & A(t) \cos \left(\omega_{0} t+\varphi(t)\right)= \\
& \operatorname{Re}\left\{A(t) \cdot \exp \left[j \cdot\left(\omega_{0} t+\varphi(t)\right)\right]\right\}
\end{aligned}
$$

where $A(t)$ and $\varphi(t)$ - are amplitude and phase that vary slowly with respect to carrier (amplitude and phase modulation), $\omega_{0}-$ is the carrier frequency. Its complex envelope is

$$
\overline{A(t)}=A(t) \cdot \exp [j \varphi(t))]
$$

So, there is not any carrier information in the complex envelope, only modulation information (only the first harmonic zone is taken into account). It's very important from the viewpoint of computational efficiency, but it also limits the technique capabilities - only narrowband analysis is possible because the frequency response is assumed to be flat over the simulation bandwidth. The output signal of a bandpass nonlinear stage is

$$
\begin{aligned}
& y(t)=K\left(A_{\text {in }}(t)\right) \cdot A_{\text {in }}(t) \times \\
& \quad \cos \left(\omega_{0} t+\varphi_{\text {in }}(t)+\Phi\left(A_{\text {in }}(t)\right)\right)
\end{aligned}
$$

where $A_{\text {in }}$ and $\varphi_{\text {in }}$ are the input signal amplitude and phase. A nonlinear stage is characterized by its envelope amplitude and phase transfer factors:

$$
K\left(A_{\text {in }}\right)=\frac{A_{\text {out }}}{A_{\text {in }}}, \quad \Phi\left(A_{\text {in }}\right)=\varphi_{\text {out }}-\varphi_{\text {in }}
$$

$K\left(A_{\text {in }}\right)$ represents envelope amplitude-to-amplitude (AM-AM) nonlinearity, and $\Phi\left(A_{\text {in }}\right)$ represents envelope amplitude-tophase (AM-PM) nonlinearity. Note that both factors depend on the input signal amplitude, not on instantaneous value of the signal. It's due to the bandpass representation of signals and system stages (actually, lowpass equivalents of both are used). Thus, equation (4) constitutes the envelope nonlinearity.

In the quadrature modelling technique, in-phase and quadrature envelope transfer factors are used

$$
\begin{aligned}
& K_{I}\left(A_{\text {in }}\right)=K\left(A_{\text {in }}\right) \cos \Phi\left(A_{\text {in }}\right) \\
& K_{Q}\left(A_{\text {in }}\right)=K\left(A_{\text {in }}\right) \sin \Phi\left(A_{\text {in }}\right)
\end{aligned}
$$

and output lowpass signal is expressed as

$$
Y(t)=K_{I}\left(A_{\text {in }}\right) X_{I}(t)-K_{Q}\left(A_{\text {in }}\right) X_{Q}(t)
$$

Two independent channels (in-phase (I) and quadrature (Q)) are used for the simulation. In this way this technique takes into account both the AM-AM and AM-PM nonlinearities. This nonlinear model is sometimes called a memoryless nonlinearity in the sense that the transfer factors $K_{I}$ and $K_{Q}$ depend on the input signal amplitude $A_{\text {in }}$ at the same instant only.

At the present time, this technique is mainly used for the simulation of solid-state power amplifiers. It has many advantages: it allows one to simulate the power amplifier with a digitally-modulated input signal using a PC in reasonable time and, consequently, to predict ACPR, power spectral regrowth (PSR) and EVM. The technique can also predict IMPs and gain compression/expansion. The permissible number of input tones and the analysis dynamic range are quite large. However, the main drawback of the quadrature modeling technique is that it is a narrowband one, so it can not take into account frequency response, to predict harmonics of the carrier frequency and even-order nonlinear products, or to model the bias decoupling network effect [5]. This effect limits the analysis accuracy even for narrowband signals and systems. The quadrature modeling technique also uses an explicit representation of the modulated signal, so multiple-carrier signals can not be simulated in a direct way. Thus, some improvements are desirable. 


\section{INSTANTANEOUS QUADRATURE TECHNIQUE}

The main idea behind the instantaneous quadrature technique is to combine the quadrature modeling technique and the discrete technique (its detailed description can be found in $[7,8]$ ) in order to build a simulation technique which can model the circuit or system behavior over wide frequency and dynamic ranges taking into account both AM-AM and AMPM conversions [5,6]. In order to model signals and systems over a wide frequency range, the instantaneous values of the signals must be used, not the complex envelope. In order to model the AM-PM conversion, the quadrature modeling structure should be used for the nonlinear element modeling. Thus, the modeling process consists of the following items:

1. Linear filters are modeled in the frequency domain (the same as for the discrete technique).

2. Nonlinear elements are modeled in the time domain using the quadrature structure, but the instantaneous signal values are used, not the complex envelope.

3. The transform from the frequency (time) domain to the time (frequency) domain is made by IFFT (FFT) (very computationally efficient).

4. The Hilbert transform is used to calculate in-phase and quadrature components,

$$
x_{Q}(t)=\hat{x}(t)=\int_{-\infty}^{\infty} x(\tau)(t-\tau)^{-1} d \tau, x_{I}(t)=x(t),
$$

where $x_{I}(t)$ and $x_{Q}(t)$ are instantaneous in-phase and quadrature components of the signal, $\hat{x}(t)$ is the Hilbert-conjugate signal of $x(t)$. In fact, we use the Hilbert Transform in the frequency domain to calculate the quadrature components because it does not require numerical integration and, thus, is much more computationally efficient: $x_{Q}(t)=\operatorname{IFFT}(-j \operatorname{sgn}(\omega) S(\omega))$, where $S(\omega)=F F T(x(t))$ is the signal's spectrum. The signal itself is the in-phase component, and the Hilbert's conjugate signal is the quadrature component.

A system of two integral equations is used in order to convert the envelope transfer function into the instantaneous ones:

$$
\begin{aligned}
& \frac{4}{\pi} \int_{0}^{1} k_{I}\left(A_{\text {in }} t\right) \frac{t^{2} d t}{\sqrt{1-t^{2}}}=K\left(A_{\text {in }}\right) \cos \Phi\left(A_{\text {in }}\right), \\
& \frac{4}{\pi} \int_{0}^{1} k_{Q}\left(A_{\text {in }} t\right) \sqrt{1-t^{2}} d t=K\left(A_{\text {in }}\right) \sin \Phi\left(A_{\text {in }}\right)
\end{aligned}
$$

where $k_{I}$ and $k_{Q}$ are the instantaneous in-phase and quadrature transfer factors. Note that using (8) only the even parts of the transfer factors can be calculated. In order to find the odd parts, some additional characteristics should be used (for instance, the second harmonic transfer factor). As a rule, the amplitude transfer characteristics can be measured or simulated using a circuit-level simulator, thus we need to solve equations (8) for $k_{I}$ and $k_{Q}$. This can be done using the method of moments. If we use piecewise constant basis functions and a point matching technique, the matrices of these equations appear to be upper triangular ones, so the systems of linear equations can be solved analytically (this semi-analytical approach speeds up computations substantially). Fig. 1 gives an illustration of the nonlinear element modelling using this approach.

If one needs to simulate even-order nonlinear products, which may be of considerable interest for broadband systems, the odd parts of the instantaneous transfer factors have to be determined. It can be done using the second-order envelope characteristics (second harmonic zone AM-AM and AM-PM functions or second-order IMP at the output for the two-tone input):

$$
\begin{aligned}
& \frac{4}{\pi} \int_{0}^{1} k_{I}\left(A_{\text {in }} t\right) \frac{t\left(2 t^{2}-1\right)}{\sqrt{1-t^{2}}} d t=K_{2}\left(A_{\text {in }}\right) \cos \left(\Phi_{2}\left(A_{\text {in }}\right)\right) \\
& \frac{8}{\pi} \int_{0}^{1} k_{Q}\left(A_{\text {in }} t\right) t \sqrt{1-t^{2}} d t=K_{2}\left(A_{\text {in }}\right) \sin \left(\Phi_{2}\left(A_{\text {in }}\right)\right)
\end{aligned}
$$

where $K_{2}\left(A_{\text {in }}\right)$ is the second-order envelope transfer factor and $\Phi_{2}\left(A_{\text {in }}\right)$ is the second-order AM-PM characteristic. We can solve the integral equations (9) using the traditional method of moments approach. But, as practical experience shows, it requires even more sample points than for the even parts. Thus, it is much efficient to use the semi-analytical approach proposed above.

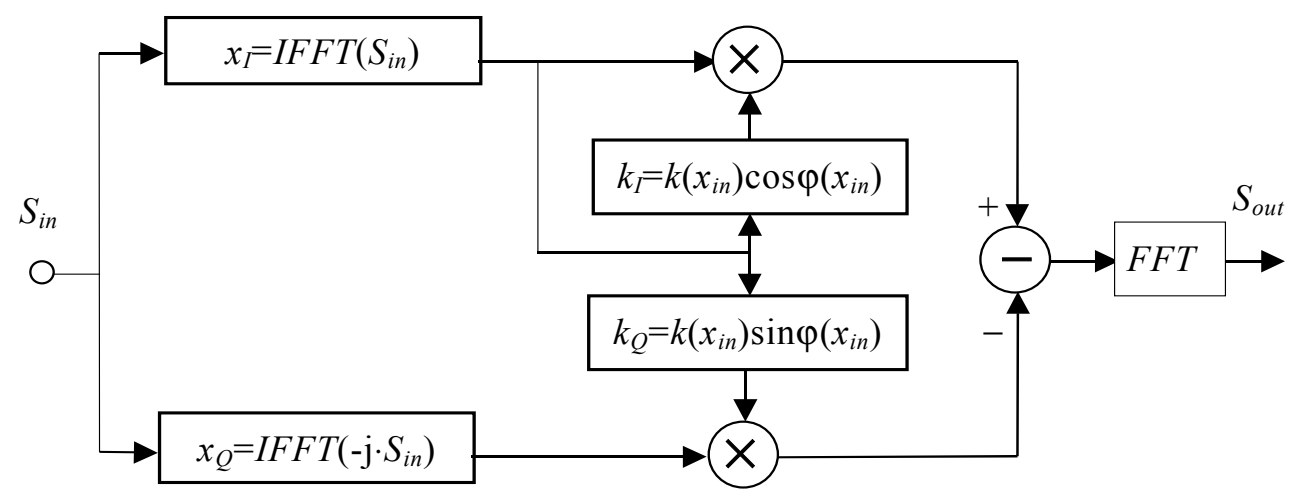

Figure 1. Numerical model of a broadband nonlinear element in the instantaneous quadrature technique. The input and output signals are in the frequency domain. 


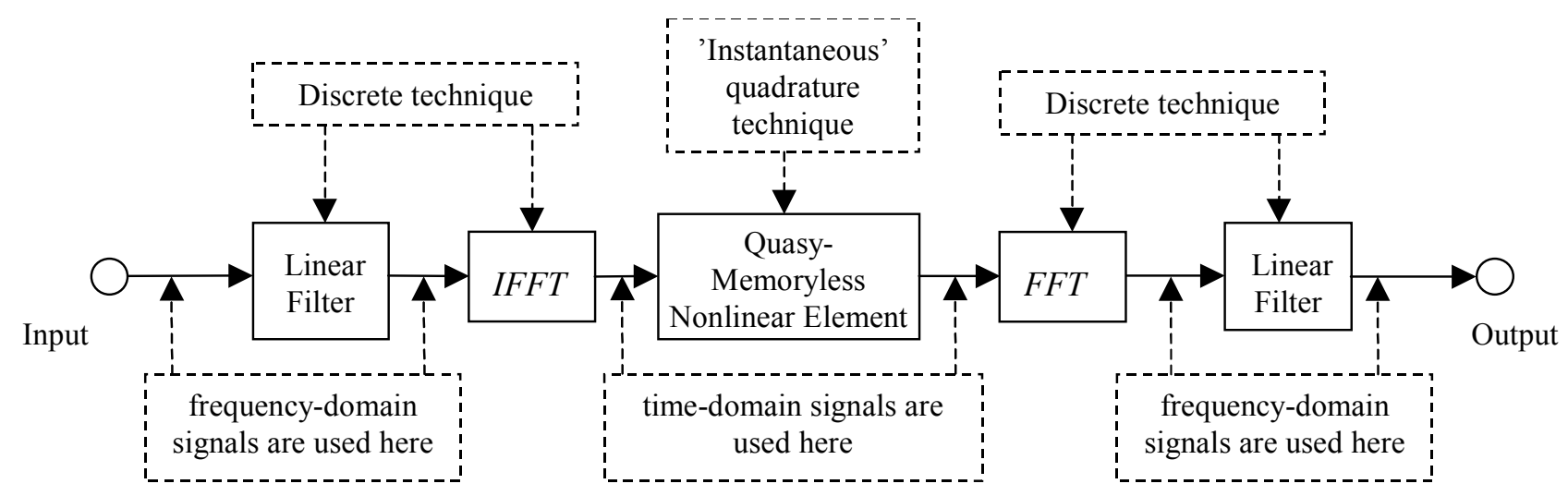

Figure 2. Simulating a single-stage RF amplifier (including the matching networks) using the instantaneous quadrature technique.

Analytical models of the instantaneous transfer factor can also be used [9-11]. However, care should be taken when designing such models to make sure that all the derivatives exists (i.e., the model should be "smooth" enough) since otherwise incorrect results can be obtained [12].

Let us now consider the simulation of typical wireless subsystems/systems using the instantaneous quadrature technique. The simulation of a single stage RF amplifier (either power or low noise) is straightforward: linear filters model the matching networks, and the quasy-memoryless nonlinear elements model the amplifier nonlinearity (both AM-AM and AM-PM). Using this approach, we may simulate other RF and IF stages (like mixers and IF amplifiers) and even detectors [6] in a computationally-efficient way. Further, using the black-box approach, we may simulate the entire link and network operation under real-world conditions and signals. The simulation of a wireless transmitter using the instantaneous quadrature technique is quite straight-forward: we do it in the same way as for the quadrature modeling technique. The power amplifier is considered to be the main source of interference and distortions in this case (however, other stages can also be simulated).

The simulation of a wireless receiver is not so simple because many its components can contribute to the overall interference and distortions. Thus, we consider (i.e. simulate) the receiver block diagram step by step: low-noise amplifier, local oscillator plus mixer, IF filter and IF amplifier, detector and baseband signal processing part. It should be noted that (i) frequency response is taken into account in every stage, and (ii) detector simulation is possible using the technique proposed that enables us to go further to the simulation of baseband signal processing. DSP can be simulated using either the traditional approach or the instantaneous quadrature technique. Thus, the instantaneous quadrature technique can be used for the EMC/EMI simulation of the entire wireless communication link over wide frequency and dynamic ranges.

The technique has been demonstrated to be able to predict accurately the nonlinear performance of RF amplifiers (i.e., IMPs, harmonics, desensitization etc.) over wide frequency and dynamic ranges in a computationally-efficient way [4], so the simulation of digital systems is also possible. In order to validate the instantaneous quadrature technique, extensive harmonic-balance simulations as well as measurements of microwave solid-state amplifiers have been carried out [5]. Fig. 3 shows IMPs simulated by the instantaneous quadrature technique (solid line) and by the harmonic balance technique (squares). One can note quite a good agreement between behavioral-level and harmonic balance (circuit level) simulations. In general, the discrepancy is about few dBs except for some special areas. Fig.4 shows harmonics simulated by the instantaneous quadrature technique (solid line) and measured (squares). Behavioral-level simulation and measurements agree quite well in this case as well. Note also that the analysis is made over wide dynamic range (130-180 $\mathrm{dB}$ ) and wide frequency range (harmonics!). Thus, these results seem to be very satisfactory taking into account that the problem is a nonlinear one. Accuracy of even-order nonlinear products prediction is slightly worse but still satisfactory for practical purposes.

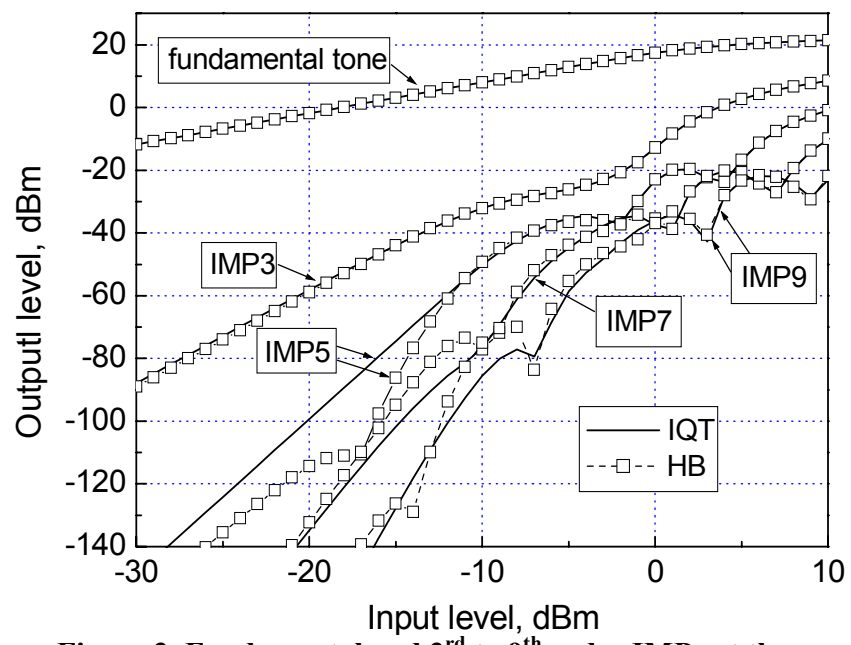

Figure 3. Fundamental and $3^{\text {rd }}$ to $9^{\text {th }}$ order IMPs at the microwave amplifier output (solid line - instantaneous quadrature technique, squares - harmonic balance (HB) technique). 


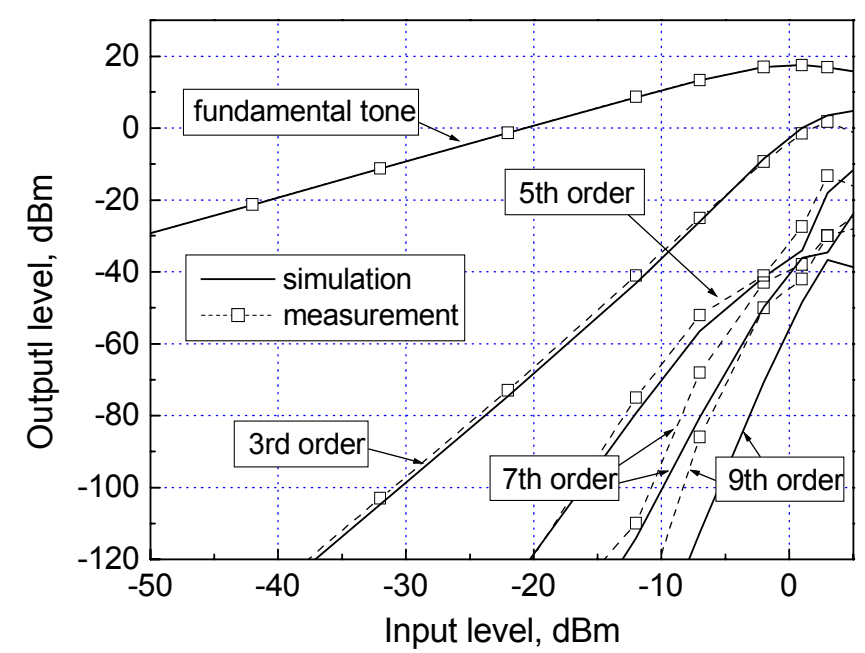

Figure 4. Fundamental and $3^{\text {rd }}$ to $9^{\text {th }}$ harmonics (solid line simulated, squares - measured) for two-stage MMIC amplifier.

It should be noted that the instantaneous quadrature technique can be easily combined with the linear simulation techniques, which predict the performance of baseband signal processing [9-11]. However, much work remains to be done before practical and efficient simulation (software) tool will be available.

\section{REFERENCES}

[1] J.H. Winters, Smart Antennas for Wireless Systems, IEEE Personal Communications, Feb. 1998, pp. 23-27.

[2] M. Shafi et al (Ed.), Wireless Communications in the $21 \mathrm{st}$ Century, Wiley \& IEEE Press, 2002.
[3] S.L. Loyka, "The Influence of Electromagnetic Environment on Operation of Active Array Antennas: Analysis and Simulation Techniques," IEEE AP Magazine, Dec. 1999, vol. 41, N 6, pp. 23 39.

[4] D. Gesbert et al, From theory to practice: an overview of MIMO space-time coded wireless systems, IEEE JSAC, v. 21, N. 3, pp. 281302 , Apr 2003.

[5] S. Loyka, J.R. Mosig, "New behavioral-level simulation techniques for RF/microwave applications. Part I: Basic concepts \& Part II: Approximation of nonlinear transfer functions ", Int. Journal of RF and Microwave Computer-Aided Engineering, vol. 10, no. 4, July 2000, pp. 221-237 \& pp. 238-252.

[6] S.L. Loyka, J.R. Mosig, New Behavioral-Level Simulation Technique for RF/Microwave Applications. Part III: Advanced Concepts, International Journal of RF and Microwave ComputerAided Engineering, v. 12, N.2, pp.206-216, Mar. 2002.

[7] V.I. Mordachev "Express analysis of electromagnetic compatibility of radio electronic equipment with the use of the discrete models of interference and Fast Fourier Transform", Proc. of IX Inter. Wroclaw Symp. on EMC, Poland, Wroclaw, 1988, Part 2, pp.565-570.

[8] S. L. Loyka and V. I. Mordachev, "Identification of Nonlinear Interference Sources with the use of the discrete technique," IEEE EMC Symp., Denver, Colorado, Aug. 24-28, 1998, pp. $882-887$.

[9] M.C. Jeruchim, P. Balaban, K.S. Shanmugan, Simulation of Communication Systems, Kluwer, New York, 2000.

[10] W. H. Tranter et al, Principles of Communication System Simulation, Prentice Hall, Upper Saddle River, NJ, 2004.

[11] F.M. Gardner, J.D. Baker, Simulation Techniques, Wiley, New York, 1997.

[12] S.L. Loyka, On the Use of Cann's Model for Nonlinear Behavioral-Level Simulation, IEEE Trans. on Vehicular Technology, vol. 49, N. 5, pp. 1982-1985, Sep. 2000. 